\title{
Pulmonary actinomycosis diagnosed by transbronchoscopic lung biopsy: A case report and literature review
}

\author{
XING DING $^{1}$, GENGYUN SUN $^{1}$, GUANGHE FEI $^{1}$, XUEXIN ZHOU $^{2}$, LUQIAN ZHOU $^{2}$ and RAN WANG ${ }^{1}$ \\ ${ }^{1}$ Department of Respiratory and Critical Care Medicine, The First Affiliated Hospital of Anhui Medical University, \\ Hefei, Anhui 230022; ${ }^{2}$ The First Clinical College of Anhui Medical University, Hefei, Anhui 230001, P.R. China
}

Received February 8, 2018; Accepted July 2, 2018

DOI: $10.3892 /$ etm.2018.6483

\begin{abstract}
Pulmonary actinomycosis is a chronic, suppurative, granulomatous disease caused by Actinomyces israelii, an obligate anaerobe. The clinical manifestations and imaging characteristics of pulmonary actinomycosis lack specificity and can lead to confusion with tuberculosis and lung cancer. The present study reported a case of pulmonary actinomycosis diagnosed by transbronchoscopic lung biopsy and reviewed the literature on the disease. The clinical characteristics, signs, laboratory findings as well as progression, diagnosis and treatment in the case of pulmonary actinomycosis were analyzed. The patient was diagnosed by transbronchoscopic lung biopsy. After two weeks of antibiotic therapy, the cough was significantly improved and the patient's temperature returned to normal. Moreover, the lesion in the left lower lung was significantly smaller. Pulmonary actinomycosis is usually confused for tuberculosis and lung cancer. The present findings indicated that transbronchoscopic lung biopsy is a useful tool for diagnosing the disease. To conclude, doctors should have a clear enough understanding of the disease to prescribe empirical antibiotics and avoid unnecessary surgery.
\end{abstract}

\section{Introduction}

Actinomyces is a non-infectious and facultative, anaerobic, gram-positive bacterium, which has a filamentous shape and is characterized by slow growth. It is commonly found in the oral cavity, dental caries, and tonsils. Pulmonary actinomycosis $(1,2)$ is a chronic, suppurative, granulomatous disease caused by Actinomyces israelii, an obligate anaerobe. Most infections are due to poor oral hygiene and inhalation of secretions containing Actinomyces (3-5). Infection can also spread directly through the blood or an abdominal lesion.

Correspondence to: Dr Ran Wang, Department of Respiratory and Critical Care Medicine, The First Affiliated Hospital of Anhui Medical University, 218 Jixi Road, Hefei, Anhui 230022, P.R. China E-mail: ranwangtjmu@hotmail.com

Key words: pulmonary actinomycosis, diagnosis, treatment, transbronchoscopic lung biopsy, antibiotic
The clinical manifestations and imaging characteristics of pulmonary actinomycosis lack specificity, and can lead to confusion with tuberculosis and lung cancer. This may lead to misdiagnosis of the disease, and hence, incorrect treatment. In this report, a rare case of pulmonary actinomycosis diagnosed by transbronchoscopic lung biopsy is discussed to improve the understanding of pulmonary actinomycosis and to help prevent unnecessary surgeries.

\section{Case report}

A 70-year-old man was admitted to hospital with an 'intermittent cough'. His major clinical manifestations included cough with expectoration, white phlegm, and a fever of up to $38.5^{\circ} \mathrm{C}$. The patient lacked chest congestion and asthma and there was no obvious cause for the intermittent dry cough. Chest X-ray showed pneumonia and left-sided pleural effusion, for which the patient received treatment at the local hospital. The patient had a 40-year history of smoking. The physical examination findings were generally normal. Laboratory testing revealed a white blood cell count of $16,960 / \mathrm{mm}^{3}$, hemoglobin level of $14.0 \mathrm{~g} / \mathrm{dl}$, platelet count of 296x109/1, C-reactive protein level of $21.00 \mathrm{mmol} / \mathrm{l}$, and an erythrocyte sedimentation rate of $7 \mathrm{~mm} / \mathrm{h}$. The G'GM test, and procalcitonin levels were within the reference ranges. The tumor marker concentrations were all normal (carcinoembryonic antigen $3.8 \mathrm{ng} / \mathrm{ml}$, neuron specific enolase $8.91 \mathrm{ng} / \mathrm{ml}$, CYFRA21-1 $1.25 \mathrm{ng} / \mathrm{ml}$ ). Quantification of the mycobacterium tuberculosis (TB) DNA yielded a negative test result. Sputum cultures tested negative for bacteria, fungi, and mycobacteria. Other tests were negative for the cryptococcal capsule antigen, influenza A virus antigen (swab), and evidence of Mycobacterium tuberculosis. Pleural effusion was detected and serum biochemistry results did not indicate a tumor; however, results of the lactic dehydrogenase (2009 U/l) were positive and adenosine deaminase (29.10 U/l) assay were negative. Computed tomography (CT) revealed left-sided pleural effusion and a mass in the lower left lung (Fig. 1A and B).

The combination of the history and laboratory test results did not rule out lung cancer. However, the patient had a cough and evidence of inflammation, which suggested the presence of lung infection. The patient was treated with levofloxacin $(0.4 \mathrm{~g}$ ivgtt $\mathrm{qd})$ and mezlocillin sodium/sulbactam sodium (3.75 mg ivgtt q $8 \mathrm{~h}$ ). At the same time, bronchoscopy was 


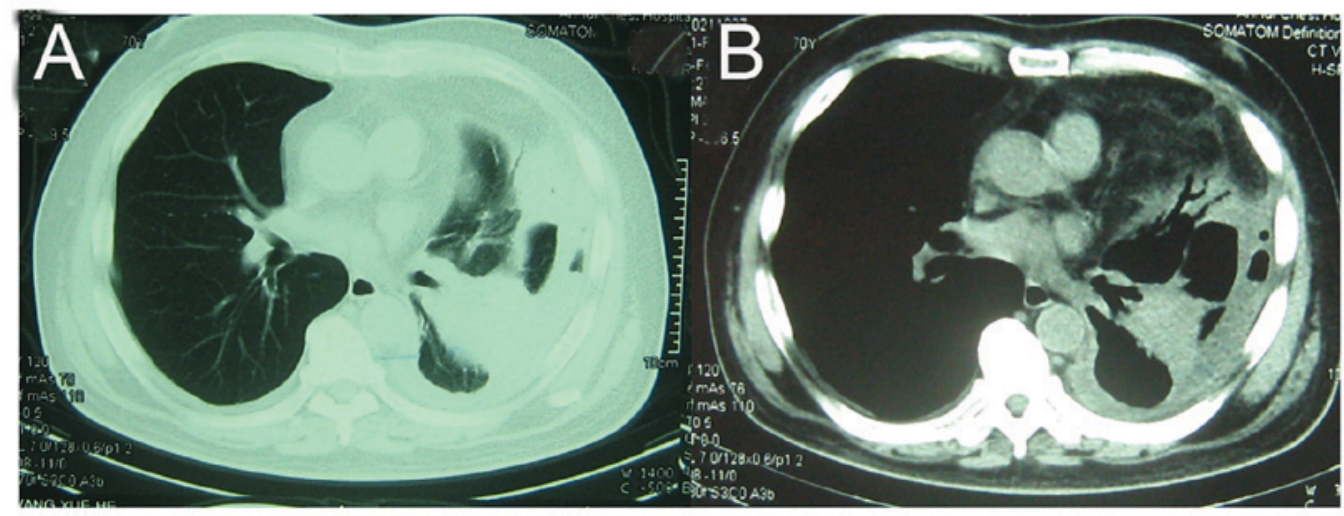

Figure 1. Computed tomography (CT) revealed left-sided pleural effusion and a mass in the lower left lung. (A) Lung window; (B) Mediastinum window.

performed, which suggested the presence of a white, necrotic neoplasm obstructing the lumen of the dorsal segment of the lower lung lobe (Fig. 2). After treatment with antibiotics and thoracocentesis, the cough and other symptoms improved slightly, but the fever persisted. Furthermore, histopathologic examination showed a large number of lymphocytes, neutrophils, and plasma cells in the bronchial mucosal tissues. In addition, we also identified some thin filaments and floccus surrounded by areas of necrosis, which stained positive with hexamine-silver. Therefore, we determined actinomycosis as the cause of this patient's symptoms (Fig. 3). After two weeks of antibiotic therapy, the cough was significantly improved and the patient's temperature had returned to normal. We therefore repeated the $\mathrm{CT}$ scan and found that the lesion in the left lower lung was significantly smaller (Fig. 4A and B). It was recommended that the patient be treated with antibiotics for an additional 3 months after being discharged from the hospital. The present study was approved by the Ethics Committee of Anhui Medical University (Hefei, China) and informed consent was obtained from the patient.

\section{Discussion}

Actinomyces was the first actinomycete to be discovered in 1899 by Bujuid as part of the normal flora in the human mouth. Actinomyces are bacteria, not fungi, and are characterized by the formation of multiple abscesses, sinuses, and abundant granulation tissue $(6,7)$. Their main reproductive method is fission rather than budding, and because there is no sterol in the cell wall, they are resistant to many antifungal drugs but are sensitive to penicillin.

Pulmonary actinomycosis $(8-10)$ is a rare infection caused by Actinomyces. It is a suppurative, granulomatous disease, usually caused by inhaled oropharyngeal secretions. Long-term smokers often have bad oral hygiene, which may be a factor in the development of an infection with Actinomyces. In addition, some underlying lung conditions such as emphysema, chronic bronchitis, and bronchiectasis can increase the incidence of Actinomyces infection. There are reports $(5,9,11,12)$ that link pulmonary actinomycosis with alcoholism, invasive screening, poor oral hygiene, and various chronic conditions (e.g., diabetes, immunosuppressive disease, viral hepatitis, malnutrition). When the body's resistance declines, due to the oral secretions that have been inhaled into the respiratory tract,

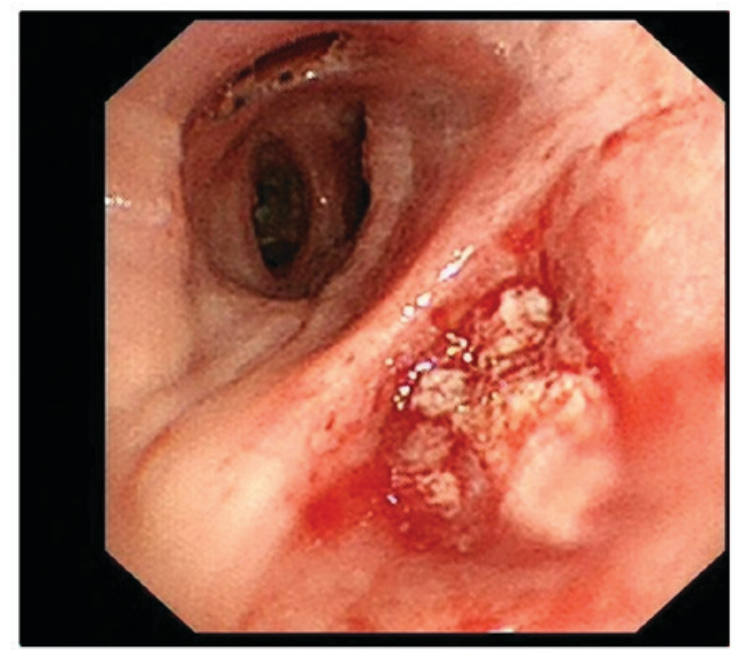

Figure 2. Bronchoscopy showed that a white, necrotic neoplasm obstructing the lumen of the dorsal segment of left lower lung lobe (magnification, $\mathrm{x} 4$ ).

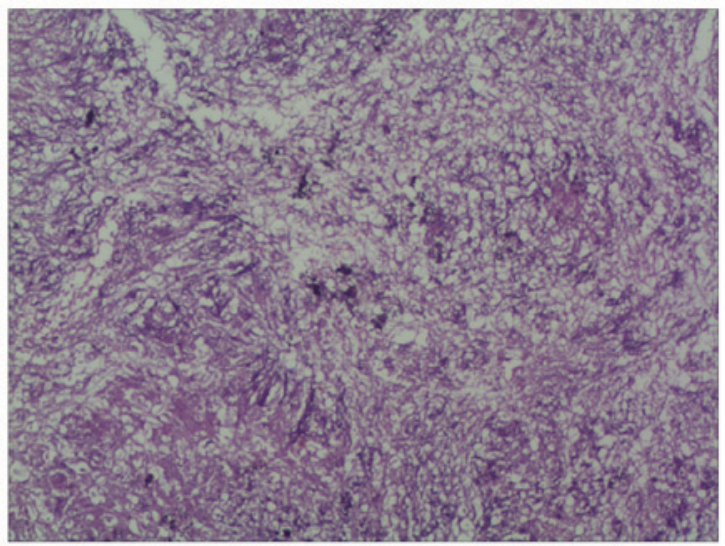

Figure 3. Histopathologic examination showed a large number of lymphocytes, neutrophils, and plasma cells in the bronchial mucosal tissues.

bronchial lesions develop that may spread to the mediastinum. Esophageal lesions caused by suppurative pneumonia, blade clearance, and invasion of the chest wall, ribs, and antrum, can all result in invasion of the circulation and subsequent systemic spread. Actinomycosis can occur at any age, but occurs primarily in young or middle-aged people (20-50 years old). 


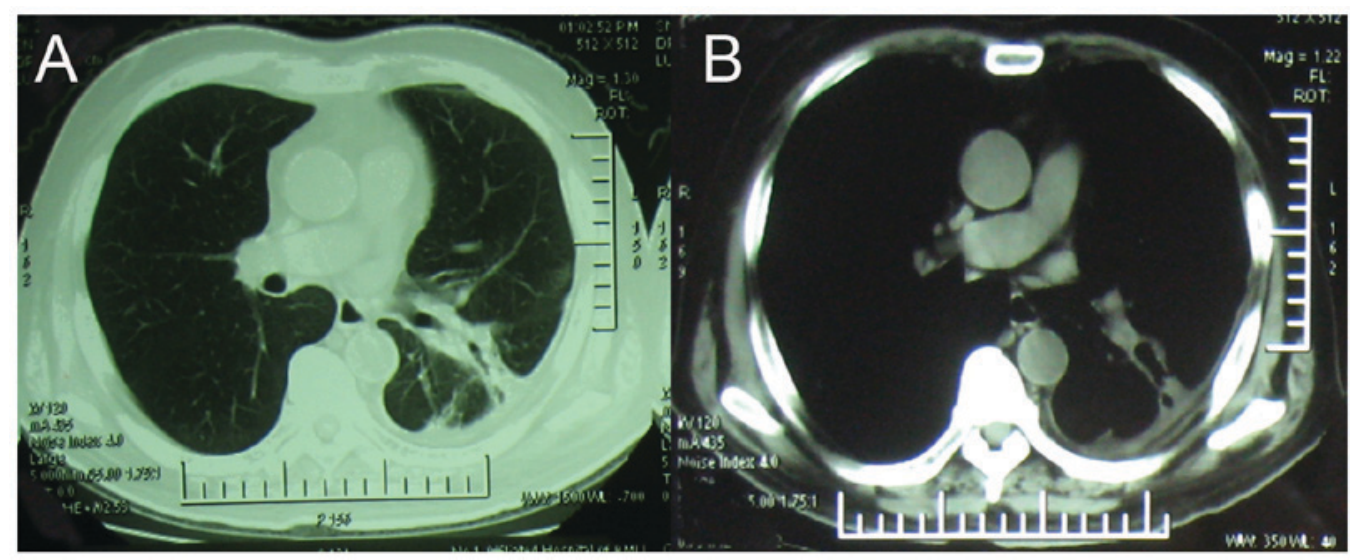

Figure 4. Computed tomography (CT) revealed that lesion in the left lower lung was significantly smaller. (A) Lung window; (B) Mediastinum window.

In addition, the incidence of actinomycosis in men is two to four times as high as it is in women, which may be associated with a higher incidence of poor oral health and facial injuries in men (13). In this case, a 70-year-old senile male, with a 40-year history of smoking and diabetes mellitus, produced oropharyngeal secretions that were inhaled into the lungs and ultimately led to the development of pulmonary actinomycosis.

Pulmonary actinomycosis has no specific clinical manifestation. As it progresses, there are a variety of manifestations of the disease, but there are no early symptoms. Pulmonary actinomycosis $(6,14)$ can be characterized by a low-grade fever, weight loss, fatigue, expectoration, shortness of breath, hemoptysis and/or symptoms such as chest pain and sulfur particles in the sputum $(5,9,15)$. The disease is easily confused with tuberculosis, bronchiectasis, aspergillus or other fungal infections, lung neoplasia, and pulmonary abscesses $(11,16-21)$. Research in Europe shows that the most common symptoms of pulmonary actinomycosis are coughing and chest pain. Hemoptysis is more commonly observed in Asia. In the case in this report, the patient experienced only an intermittent cough with expectoration, fever, and an elevated white blood cell count and CRP inflammation index. This corresponds to the general manifestation of pneumonia, but is not specific to the diagnosis of pulmonary actinomycosis. Pulmonary actinomycosis usually develops in the absence of segmental pneumonia. Kim et al (22) studied the images of 94 patients and reported that the features of pulmonary actinomycosis observed most frequently were low-density shadows in the central region of the lungs. The lesions mainly dominated the left lower and the right middle lung lobes (6). However, pulmonary actinomycosis is usually non-specific and can be characterized by cavitations, pleural effusion, shadowing, air bronchograms, and parenchymal masses to name a few. Of course, the above abnormalities are often misdiagnosed as neoplasia or tuberculosis $(10,23)$. In recent years, several studies have shown that many physicians misdiagnose pulmonary actinomycosis as lung cancer. Gazzoni (24) found that CT images obtained in cases of pulmonary actinomycosis are similar to those obtained in aspergillosis. On CT $(25,26)$, a hole is seen in which the mycelium of the Actinomyces coexist with the fungus. This form of actinomycosis usually occurs in diabetic patients, and it is worth noting that the cavity in question is also visible with mycobacterium infection and neoplasia. In the case reported here, the chest $\mathrm{CT}$ revealed left-sided pleural effusion and a mass effect in the left lower lung. The man had a long history of smoking, and there was no apparent cause for the intermittent dry cough. In consideration of this history, we surmised that a malignant lung tumor could not be ruled out. However, the patient had an elevated white blood cell count, which was consistent with inflammation. We prescribed empirical antibiotic therapy consisting of mezlocillin and sulbactam. After two weeks of treatment, the patient's symptoms had improved significantly and the lesion was found to be significantly smaller.

Currently, the diagnosis of pulmonary actinomycosis depends on visualization of the filaments. Because a positive sputum culture is so rare, so we often utilize bronchoscopy (14). The rate of detection of pulmonary Actinomyces using bronchoscopy was significantly lower than it was with surgical biopsy. But pulmonary actinomycosis is known to be sensitive to penicillin. The trauma associated with fibrobronchoscopy biopsy or percutaneous biopsy was preferred to surgical trauma. A number of documented clinical cases show that pulmonary actinomycosis has a high rate of misdiagnosis. Three main factors may contribute to this phenomenon. First, there is the fact that pulmonary actinomycosis is rare, and clinicians do not always recognize it. Second, the clinical manifestation and imaging characteristics of pulmonary actinomycosis are similar to those of tuberculosis and neoplasia. Third, the dissociation of Actinomyces is difficult, and sputum smear and culture has a low detection rate. In this case, the patient was diagnosed with secondary fibrobronchoscopy pathology. So clinically, the diagnosis of lung cancer could not be ruled out by a single negative bronchoscopy. Second-look bronchoscopy should be considered, especially when CT-guided lung biopsy is contraindicated. In addition, the symptoms of patients suspected of having a lung tumor and showing mass shadowing on CT images were significantly improved after the treatment with antibiotics. In this study, when the focal lesion began to shrink, the diagnoses of tuberculosis and neoplasia were excluded. Bronchoscopy is then preferred to clarify the presence of pulmonary actinomycosis.

The current standard of care for the treatment of pulmonary actinomycosis includes antimicrobial therapy with or without surgery. The main therapy consists of high-dose intravenous penicillin for 2-6 weeks (generally $18-24 \times 10^{6}$ units of penicillin per day), followed by oral penicillin $\mathrm{V}$ (or amoxicillin) 
for an additional 6-12 months $(6,15)$. Previous research has indicated that long-term penicillin use can reduce the risk of recurrence. Nevertheless, those with allergies to penicillin may consider the use of macrolide antibiotics (e.g., erythromycin) and first-generation cephalosporins. Pulmonary actinomycosis may also be combined with other bacterial infections. When penicillin alone is not effective, it can be combined with an aminoglycoside as part of a broad-spectrum antimicrobial coverage. At present, the clinical diagnosis of pulmonary actinomycosis is difficult, and requires that doctors have a clear enough understanding of the disease to prescribe empirical antibiotics and avoid unnecessary surgery.

Pulmonary actinomycosis is difficult to distinguish from tuberculosis and neoplasia both clinically and radiographically. Therefore, the presence of pulmonary actinomycosis should be considered in patients who are suspected of having tuberculosis or neoplasia. Early diagnosis and treatment of pulmonary actinomycosis is crucial (27). The prognosis for this disease is good when treated appropriately, and its proper diagnosis prevents unnecessary surgery. Therefore, it is vital that every clinician be proficient in the diagnosis and treatment of pulmonary actinomycosis.

\section{Acknowledgements}

The authors thank the patient and her parents for their cooperation during the diagnostic process. This research was supported by the fund from the first affiliated hospital of Anhui Medical University for reserve talents (2014), the fund for Excellent top talent cultivation project of Anhui Higher Education Institutions (gxyqZD2017030), the fund from quality engineering project of education department of Anhui province (2015jyxm780, 2016jtxx093).

\section{Funding}

The present study was supported by funding from the First Affiliated Hospital of Anhui Medical University for Reserve Talents (2014), the Excellent Top Talent Cultivation Project of Anhui Higher Education Institutions (grant no. gxyqZD2017030), the Quality Engineering Project of Education Department of Anhui Province (grant nos. 2015jyxm78 and 2016jtxx093).

\section{Availability of data and materials}

The analyzed datasets generated during the study are available from the corresponding author on reasonable request.

\section{Authors' contributions}

RW designed the study and wrote the paper. XD, GS, GF, XZ, LZ collected the data. XD, GS, GF revised the manuscript. All authors read and approved the final manuscript.

\section{Ethics approval and consent to participate}

Written informed consent was obtained from the patient in the present study. The study was approved by the Ethics Review Board at Anhui Medical University.

\section{Patient consent for publication}

The patient provided written informed consent for the publication of their data.

\section{Competing interests}

The authors declare that there were no competing interests.

\section{References}

1. Boyanova L, Kolarov R, Mateva L, Markovska R and Mitov I: Actinomycosis: A frequently forgotten disease. Future Microbiol 10: 613-628, 2015.

2. Wong VK, Turmezei TD and Weston VC: Actinomycosis. BMJ 343: d6099, 2011.

3. Cendan I, Klapholz A and Talavera W: Pulmonary actinomycosis. A cause of endobronchial disease in a patient with AIDS. Chest 103: 1886-1887, 1993.

4. Gupta P, Dogra V, Goel N, Chowdhary A, Prasad R and Gaur SN: An unusual cause of a pulmonary mass: Actinomycosis. Indian J Chest Dis Allied Sci 57: 177-179, 2015.

5. Jehangir W, Vaidya B, Enakuaa S, Raoof N, Middleton JR and Yousif A: Thoracic actinomycosis: A rare occurrence. Infect Dis Rep 8: 5963, 2016.

6. Mabeza GF and Macfarlane J: Pulmonary actinomycosis. Eur Respir J 21: 545-551, 2003.

7. Moskowitz SM, Shailam R and Mark EJ: Case records of the massachusetts general hospital. Case 25-2015. An 8-year-old girl with a chest-wall mass and a pleural effusion. New Engl J Med 373: 657-667, 2015.

8. Farrokh D, Rezaitalab F and Bakhshoudeh B: Pulmonary actinomycosis with endobronchial involvement: A case report and literature review. Tanaffos 13: 52-56, 2014.

9. Mani RK, Mishra V, Singh PK and Pradhan D: Pulmonary actinomycosis: A clinical surprise! BMJ Case Rep 2017: pii: bcr2016218959, 2017.

10. Park JY, Lee T, Lee H, Lim HJ, Lee J, Park JS, Cho YJ, Park YS, Lee $\mathrm{CH}$, Lee SM, et al: Multivariate analysis of prognostic factors in patients with pulmonary actinomycosis. BMC Infect Dis 14: 10, 2014.

11. Katsenos S, Galinos I, Styliara P, Galanopoulou N and Psathakis K: Primary bronchopulmonary actinomycosis masquerading as lung cancer: Apropos of two cases and literature review. Case Rep Infect Dis 2015: 609637, 2015.

12. Thomas M, Raza T and Langawi MA: A 37-year-old man with nonresolving pneumonia and endobronchial lesion. Chest 148: e52-e 55, 2015 .

13. Han JY, Lee KN, Lee JK, Kim YH, Choi SJ, Jeong YJ, Roh MS and Choi PJ: An overview of thoracic actinomycosis: CT features. Insights Imaging 4: 245-252, 2013.

14. Skehan N, Naeem M and Reddy RV: Endobronchial actinomycosis: Successful treatment with oral antibiotics. BMJ Case Rep 2015: pii: bcr2015212754, 2015.

15. Sobajima T, Asano F, Tsuzuku A, Murakami A, Masuda A, Matsuno Y and Matsumoto S: A case of pulmonary actinomycosis associated with aspiration of cedar leaves. J Bronchology Interv Pulmonol 22: 259-262, 2015.

16. Atay S, Banki F and Floyd C: Empyema necessitans caused by actinomycosis: A case report. Int J Surg Case Rep 23: 182-185, 2016.

17. Brook I: Actinomycosis: Diagnosis and management. South Med J 101: 1019-1023, 2008.

18. Choi J, Koh WJ, Kim TS, Lee KS, Han J, Kim H and Kwon OJ: Optimal duration of IV and oral antibiotics in the treatment of thoracic actinomycosis. Chest 128: 2211-2217, 2005.

19. Lionakis MS and Hamill RJ: Malaise, weight loss, pleuritic chest pain and productive cough: What is your call? CMAJ 178: 1289-1291, 2008

20. Song JU, Park HY, Jeon K, Um SW, Kwon OJ and Koh WJ: Treatment of thoracic actinomycosis: A retrospective analysis of 40 patients. Ann Thorac Med 5: 80-85, 2010.

21. Tsubochi H, Endo S, Suhara K and Sohara Y: Endobronchial aspergillosis and actinomycosis associated with broncholithiasis. Eur J Cardiothorac Surg 31: 1144-1146, 2007.

22. Kim SR, Jung LY, Oh IJ, Kim YC, Shin KC, Lee MK, Yang SH, Park HS, Kim MK, Kwak JY, et al: Pulmonary actinomycosis during the first decade of 21 st century: Cases of 94 patients. BMC Infect Dis 13: 216, 2013. 
23. Valour F, Sénéchal A, Dupieux C, Karsenty J, Lustig S Breton P, Gleizal A, Boussel L, Laurent F, Braun E, et al Actinomycosis: Etiology, clinical features, diagnosis, treatment, and management. Infect Drug Resist 7: 183-197, 2014.

24. Gazzoni FF, Severo LC, Marchiori E, Guimarães MD, Garcia TS, Irion KL, Camargo JJ, Felicetti JC, de Mattos Oliveira F and Hochhegger B: Pulmonary diseases with imaging findings mimicking aspergilloma. Lung 192: 347-357, 2014.

25. Costiniuk CT, Voduc N and de Souza C: Pulmonary actinomycosis in a male patient with a tracheal bronchus. Can Respir J 18 84-86, 2011.
26. Yildiz $\mathrm{O}$ and Doganay M: Actinomycoses and Nocardia pulmonary infections. Curr Opin Pulm Med 12: 228-234, 2006.

27. Chawla RK, Madan A, Chawla A and Chawla K: Hemoptysis secondary to actinomycosis: A rare presentation. Lung India 31: 168-171, 2014.

c) (i) (9) This work is licensed under a Creative Commons cc) Attribution-NonCommercial-NoDerivatives 4.0 International (CC BY-NC-ND 4.0) License. 